\title{
Socialização Parental: Adaptação ao Brasil da Escala ESPA29
}

\author{
Parental Socialization: Brazilian Adaptation of the ESPA29 Scale
}

\author{
Isabel Martínez ${ }^{*}$, a José Fernando García ${ }^{\mathrm{b}}$, Leoncio Camino ${ }^{\mathrm{c}}$ \\ $\&$ Cleonice Pereira dos Santos Camino ${ }^{c}$ \\ ${ }^{a}$ Universidad de Castilla-La Mancha, Cuenca, Espanha, ${ }^{b}$ Universidad de Valencia, Valencia, Espanha \\ \& ${ }^{c}$ Universidade Federal da Paraíba, João Pessoa, Brasil
}

\begin{abstract}
Resumo
O objetivo deste estudo foi analisar as propriedades psicométricas da versão em português brasileiro da escala de socialização parental ESPA29 (Musitu \& García, 2001). Esta escala permite medir os estilos de socialização parental - autoritativo, autoritário, indulgente e negligente - através de duas dimensões independentes da conduta parental, extraídas do uso que os pais fazem das distintas práticas de socialização. Utilizando uma amostra de 2105 adolescentes brasileiros, com idades entre os 10 e os 18 anos, replicou-se a estrutura de dois fatores - Aceitação/Implicação e Severidade/Imposição - que compõem a escala, mediante a análise fatorial exploratória. Para completar o estudo de replicabilidade, levou-se a cabo a análise da estrutura ortogonal de Procusto, comprovando-se que a estrutura era invariante para o pai e para a mãe. Palavras-chave: Estilos de Socialização; Práticas Parentais; Pontuações; Socialização; Escala de Socialização ESPA29; Adaptação.
\end{abstract}

\begin{abstract}
The objective of this study was to analyze the psychometric properties of the Brazilian version of the ESPA29 Parental Socialization Scale (Musitu \& García, 2001). This scale allows measuring parental styles - authoritative, authoritarian, indulgent, and negligent - by means of two independent dimensions of parental conduct extracted from parents' use of different socialization practices. Using a sample of 2.105 Brazilian adolescents from 10 to 18 years old, the structure of two factors that define the scale - Acceptance/Implication and Strictness/Imposition - has been replicated using an exploratory factorial analysis. In order to complete a proper replication of the ESPA29 scale structure, the analysis of the orthogonal structure of Procrustes was carried out proving that the structure was invariant for the father as well as for the mother.

Keywords: Socialization Styles; Parental Practices; Scores; Socialization; ESPA29 Socialization Scale; Adaptation.
\end{abstract}

A análise das características dos estilos de socialização parental e das suas repercussões sobre o desenvolvimento e o comportamento dos filhos constitui tradicionalmente uma das perspectivas mais relevantes no estudo das relações paterno-filiais (Arnett, 1995; Gray \& Steinberg, 1999; Maccoby \& Martin, 1983; Scarr, 1993). Os estilos de socialização se definem como padrões de comportamento persistentes dos pais face a diferentes formas de atuação dos filhos em situações da vida cotidiana (C. Camino, Camino, \& Moraes, 2003; Musitu \& García, 2001), de modo que permitem analisar uma parte importante da relação que se estabelece entre pais e filhos (Darling \& Steinberg, 1993).

Nas investigações pioneiras sobre a socialização parental (Baumrind, 1967, 1971) distinguiam-se unicamente

\footnotetext{
*Endereço para correspondência: Universidad de Castilla-La Mancha, Facultad de Ciencias de la Educación y Humanidades, Avda. de los Alfares, 44, 16071, Cuenca, Espanha. E-mails: MIsabel.Martinez@uclm.es, Fernando.Garcia@uv.es, leocamino@uol.com.br e cleocamino@yahoo.com.br
}

três estilos de socialização, autorizativo, autoritário e permissivo. Posteriormente, Maccoby e Martin (1983) propuseram quatro estilos de socialização, originados a partir de duas dimensões, teoricamente independentes, da conduta parental (Darling \& Steinberg, 1993; Smetana, 1995). Estas dimensões têm sido denominadas exigência e responsividade (e.g. Baumrind, 1991, 1996; Maccoby \& Martin, 1983; Smetana, 1995), ainda que também tenham recebido outras denominacões com significados equivalentes. As dimensões de aceitação (Symonds, 1939), implicação, (Baldwin, 1955), afeto (Becker, 1964; Sears, Maccoby, \& Levin, 1957) ou amor (Schaefer, 1959) têm significados semelhantes à denominada responsividade. Com um significado equivalente ao da dimensão exigência foram descritas as dimensões de dominação (Symonds, 1939), hostilidade (Baldwin, 1955), rigor (Sears et al., 1957), controle (Schaefer, 1959) e restrição (Becker, 1964). A dimensão de exigência refere-se à extensão na qual os pais empregam o controle, a supervisão, mantêm uma posição assertiva de autoridade para com seus filhos e exigem-lhes maturidade, ajudando 
assim a estabelecer limites à conduta do filho. Enquanto que a dimensão de responsividade representa o grau em que os pais mostram afeto e aceitação a seus filhos, dãolhes apoio e se comunicam raciocinando com eles (F. T. Costa, Teixeira, \& Gomes, 2000; Martínez \& García, 2007; Martínez, García, \& Yubero, 2007)

Diferentemente do modelo tripartido, este modelo de quatro tipos (veja-se Lamborn, Mounts, Steinberg, \& Dornbusch, 1991) enfatiza a necessidade de se levar em conta os efeitos da combinação das duas dimensões parentais na análise de sua relação com o desenvolvimento e o comportamento dos filhos. Os quatro estilos parentais que podem ocorrer, em função do grau em que estas dimensões caracterizem as atuações dos pais, são os seguintes: estilo autoritativo - caracterizado pelo emprego de alta exigência e alta responsividade por parte dos pais; estilo negligente - caracterizado por baixa exigência e baixa responsividade; estilo indulgente - baixa exigência e alta responsividade; e estilo autoritário - alta exigência e baixa responsividade. Embora os estilos autoritário e autorizativo possam considerar-se equivalentes aos que são postulados no modelo tripartido de Baumrind (1967, 1971), o estilo permissivo do antigo modelo diferencia-se, nesta nova classificação, nos estilos indulgente e negligente que, como mostram várias investigações recentes (por exemplo, García \& Gracia, 2010; Martínez, 2008, 2009; Martínez \& García, 2007, 2008; Martínez et al., 2007; Martínez \& Navarro, 2008; Musitu \& García, 2004; Villalobos, Cruz, \& Sánchez, 2004) relacionam-se de maneira muito diferente com o comportamento dos filhos.

A Escala de Socialização Parental ESPA29 (Musitu \& García, 2001) foi desenvolvida especificamente para medir os estilos de socialização utilizando uma perspectiva contextual (Darling \& Steinberg, 1993) e situacional (Oliveira et al., 2002; Smetana, 1995). Diferentemente de outros instrumentos com os quais se medem atitudes gerais, neste instrumento leva-se em conta a diferenciação assinalada por Darling e Steinberg (1993) entre práticas e estilos de socialização, de modo que os estilos se configuram a partir da utilização que os pais fazem de diversas práticas disciplinares que definem as duas dimensões da socialização, denominadas nesta escala Aceitação/Implicação e Severidade/Imposição. Para isso, os filhos devem indicar a frequência em que o pai e a mãe (considerados separadamente) empregam as várias práticas de socialização em diferentes situações representativas da vida cotidiana familiar na cultura ocidental, nas quais se representam comportamentos de obediência às normas familiares ou situações de indisciplina por parte do filho. Concretamente, analisa-se em que medida os pais empregam as práticas de afeto, indiferença, diálogo, displicência, coerção verbal, coerção física e privação.

Estas práticas parentais relacionam-se com o contexto da conduta ajustada ou desajustada do filho, com base em uma estrutura teórica aplicável para analisar varia- ções em situações gerais, não para casos clínicos graves. De maneira que, nas situações em que o filho mantém uma conduta adequada, medem-se as práticas parentais de Afeto e Indiferença, que ou reconhecem de algum modo o comportamento correto do filho ou o ignoram. Estas duas práticas devem relacionar-se negativamente entre si e, em conjunto com as práticas de Dialogo vs. Displicência, que também se relacionam negativamente entre elas, compõem a dimensão Aceitação/Implicação. As práticas de Diálogo e Displicência, por sua vez, medem-se em situações que representam contextos de desobediência à atuação parental. As práticas de Raciocínio, como o Diálogo, permitem limitar as condutas desajustadas dos filhos e relacionam-se positivamente com o reconhecimento das condutas ajustadas através do afeto (ver Figura 1), posto que ambas as práticas (diálogo e afeto) dependem, para ter lugar, de uma relação paterno-filial duradoura ótima. Por isto, se essa relação se der de maneira efetiva, refletir-se-á em pontuações altas na dimensão Aceitação/ Implicação, enquanto que pontuações baixas nesta dimensão coincidirão com relações paterno-filiais deficientes (Grusec \& Lytton, 1988; Musitu \& García, 2001, 2004; Oliva, 2006).

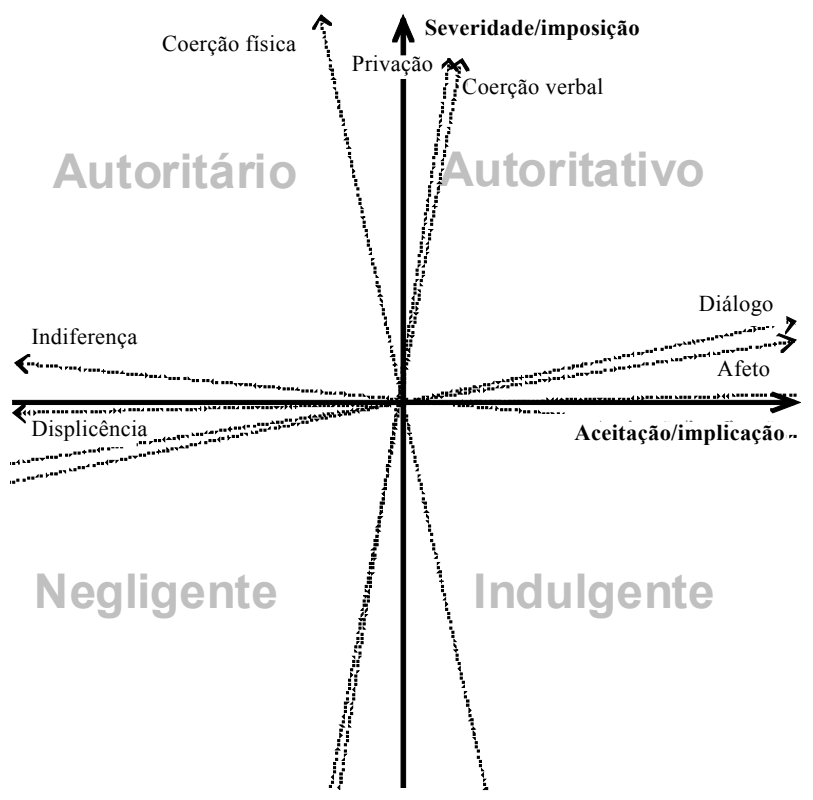

Figura 1. Modelo bidimensional de socialização e tipologias de atuação.

Por outro lado, tal como se observa na Figura 1, o emprego da coerção verbal, da coerção física e da privação relacionam-se positivamente em uma mesma dimensão, denominada, neste instrumento, Severidade/Imposição (Martínez \& Madrid, 2008). As três práticas que caracterizam esta dimensão também podem empregar-se em situações nas quais o comportamento do filho é inadequado e discrepa das normas de comportamento familiar, com o objetivo de modificar esse comportamento indisci- 
plinado, impondo restrições e limites à sua conduta de maneira que, como implica o próprio processo de socialização, possa desenvolver a habilidade para suprimir comportamentos atrativos mas proibidos e adotar outros, socialmente desejáveis (Mischel \& Mischel, 1976; Parke, 1974). No sistema de perguntas deste instrumento, não se contemplam as práticas parentais de privação, coerção verbal ou física em contextos de obediência (serviriam unicamente para tentar medir casos clínicos muito graves e, ainda assim, causariam um grande desconcerto na maioria das crianças). As práticas do eixo de Severidade/ Imposição são sistemas expeditos para inculcar as normas sociais que não dependem do grau de Aceitação/Implicação, quer dizer não se relacionam com o emprego do afeto ou a indiferença nem com o diálogo ou a displicência, por exemplo: tanto é possível que o pai raciocine com o filho depois de uma primeira reação como gritar com ele, retirar-lhe algum privilégio, quanto é possível que não o faça.

Portanto, as duas dimensões do ESPA29 são conceitualmente ortogonais e independentes de tal forma que a pontuação em uma não condiciona a pontuação na outra e, posto que as duas podem apresentar-se ou não na conduta parental em diferentes graus, é necessário contemplá-las ambas para identificar o estilo de socialização dos pais (Lamborn et al., 1991; Steinberg, Lamborn, Darling, Mounts, \& Dornbusch, 1994). Assim, o estilo autorizativo define-se por um alto emprego de práticas de Aceitação/ Implicação e de Severidade/Imposição, o estilo indulgente por alta Aceitação/Implicação e baixa Severidade/Imposição, o estilo autoritário por baixa Aceitação/Implicação e alta Severidade/Imposição e o estilo negligente por uma baixa utilização por parte dos pais tanto de práticas de Aceitação/Implicação como de Severidade/Imposição (Figura 1).
A escala ESPA29 foi originalmente validada na Espanha com uma amostra de mais de 3.000 adolescentes (Musitu \& García, 2001) de idades compreendidas entre os 10 e os 18 anos. Posteriormente foi validada na cultura italiana (Marchetti, 1997) e, recentemente, no idioma basco (López-Jáuregui \& Oliden, 2009) em uma amostra de 1.184 adolescentes. Os estilos de socialização, medidos através desta escala, foram relacionados com numerosas medidas de desenvolvimento dos filhos, como o autoconceito (Martínez \& García, 2008; Musitu \& García, 2004), os valores (Martínez \& García, 2007; Martínez \& Navarro, 2008; Moraes, C. Camino, J. B. Costa, Camino, \& Cruz, 2007), a competência pessoal, o ajuste psicológico e os problemas de conduta (García \& Gracia, 2010).

O presente trabalho tem como objetivo comprovar a estrutura fatorial do ESPA29 na população brasileira, posto que os estilos parentais são considerados independentes do contexto de socialização específico (Darling \& Steinberg, 1993) e outros instrumentos de medida identificaram as duas dimensões da socialização no Brasil (F. T. Costa et al., 2000; Martínez, 2003; Weber, Prado, Viezzer, \& Brandenburg, 2004). Espera-se replicar a estrutura fatorial obtida com a amostra original na Espanha.

\section{Método}

\section{Participantes}

A amostra brasileira utilizada para validar a adaptação foi composta de 2105 crianças e adolescentes, procedentes de uma área metropolitana do nordeste do Brasil que freqüentavam várias escolas públicas $(51,0 \%)$ e privadas $(49,9 \%)$. A percentagem de meninas representa algo mais que a metade da amostra $(54,2 \%)$ e a média de idade é de 14,9 anos $(D P=2,4)$ num intervalo de 10 a 18 anos. $\mathrm{Na}$ Tabela 1 pode-se observar a distribuição por idade e sexo da amostra deste estudo e da amostra original espanhola.

Tabela 1

Distribuição da Amostra por Idade e Sexo

\begin{tabular}{lrrrrrr}
\hline \multirow{2}{*}{ Idade } & \multicolumn{3}{c}{ Amostra Brasileira } & \multicolumn{3}{c}{ Amostra Original } \\
\cline { 2 - 6 } & Moça & Rapaz & Total & Moça & Rapaz & Total \\
\hline 10 anos & 23 & 16 & 39 & 25 & 40 & 65 \\
11 anos & 74 & 79 & 153 & 74 & 68 & 142 \\
12 anos & 91 & 90 & 181 & 149 & 145 & 294 \\
13 anos & 134 & 115 & 249 & 160 & 186 & 346 \\
14 anos & 168 & 138 & 306 & 324 & 263 & 587 \\
15 anos & 187 & 145 & 332 & 304 & 255 & 559 \\
16 anos & 141 & 144 & 285 & 281 & 236 & 517 \\
17 anos & 132 & 123 & 255 & 208 & 134 & 342 \\
18 anos & 190 & 115 & 305 & 122 & 56 & 178 \\
\hline Total & 1140 & 965 & 2105 & 1647 & 1383 & 3030 \\
\hline
\end{tabular}




\section{Instrumentos}

$\mathrm{Na}$ escala de socialização parental ESPA29 os filhos devem indicar, utilizando uma escala de 4 pontos que varia de 1 (nunca) a 4 (sempre), com que freqüência o pai e a mãe (considerados separadamente) empregam as práticas disciplinares possíveis para cada contexto. Apresentam-se vinte e nove exemplos de situações, treze das quais representam comportamentos de obediência às normas familiares por parte do filho (e. g., "Se vem alguém visitar a minha casa e comporto-me educadamente"), enquanto que nas restantes 16 apresentam-se situações de indisciplina (e. g., "Se quebro ou estrago alguma coisa de minha casa"). Nas situações que evocam comportamentos de obediência, os adolescentes indicam em que medida seus pais utilizam as práticas de Afeto ("Demonstra-me carinho") e de Indiferença ("tanto faz"), enquanto que nos itens que exemplificam comportamentos de indisciplina, indicam em que medida os pais fazem uso do Diálogo ("fala comigo") ou da Displicência ("não se preocupa comigo"), a Privação ("retira algo ou proíbe"), a Coerção verbal ("reclama comigo") e a Coerção física ("bate em mim"). Isto supõe um total de 212 respostas do filho, 106 para cada um dos pais. A pontuação na escala Aceitação/Implicação obtém-se considerando as médias das respostas nas subescalas de Afeto, Diálogo, Indiferença e Displicência para o pai e para a mãe (nas duas últimas práticas, as escalas se invertem, já que estão inversamente relacionadas com a dimensão considerada). A pontuação na escala Severidade/Imposição obtém-se considerando as médias das respostas nas subescalas de Privação, Coerção verbal e Coerção física para pais e mães. Deste modo, através das duas dimensões da conduta parental, medem-se os estilos de socialização (veja-se Lamborn et al., 1991; Steinberg et al., 1994). Os pais são classificados nos quatro tipos de socialização parental - autoritativo, indulgente, autoritário ou negligente - pelo sistema habitual de dicotomizar (Lamborn et al., 1991) as pontuações de Aceitação/Implicação e Severidade/Imposição, a partir dos tercis (Martínez \& García, 2007; Martínez et al., 2007; Martínez, Musitu, García, \& L. Camino, 2003; Musitu \& García, 2001) ou da mediana (Chao, 2001; Kremers, Brug, Vries, \& Engels, 2003), tomando-se a média das atuações do pai e da mãe. (Lamborn et al., 1991). Quando a pontuação média dos pais se situa acima da mediana ou no terceiro tercil da distribuição, nas duas dimensões, atribui-se-lhes o estilo familiar de socialização autoritativo; se estiver situada acima da mediana, ou no terceiro tercil, em Severidade/Imposição, mas abaixo da mediana ou no primeiro tercil em Aceitação/Implicação atribui-se-lhes o estilo autoritário, se estiver situada acima da mediana, ou no terceiro tercil, em Aceitação/Implicação e abaixo da mediana, ou no primeiro tercil, em Severidade/Imposição, se determina o estilo indulgente; e se a média se situa abaixo da mediana, ou no primeiro tercil, nas duas dimensões se atribui o estilo familiar negligente. As mesmas classificações podem ser feitas se- paradamente para o pai e para a mãe, caso se queira contar com essa medida.

Tradução ao Português do ESPA29. Para garantir que os itens da adaptação brasileira do ESPA29 fossem comparáveis à versão original da escala, seguiu-se o método da tradução inversa habitual (e.g., García, Musitu, \& Veiga, 2006) proposto por Brislin (1970). Depois de obter permissão dos autores, a medida inicial foi traduzida do espanhol ao português. A partir desta tradução, três investigadores da área de psicologia do desenvolvimento discutiram suas discrepâncias quanto a conteúdo, linguagem e significados. Finalmente, a medida foi de novo traduzida ao espanhol e comparada com a versão original para assegurar que os conceitos eram os mesmos.

\section{Procedimento}

Os protocolos aplicaram-se na sala de aula onde os alunos assistem coletivamente às aulas, assegurando-se que as respostas seriam anônimas. Todos os alunos contavam com a correspondente permissão parental e da direção do centro educativo. Os dados foram introduzidos em uma matriz para seu processamento no SPSS. Obtiveram-se as matrizes de padrão mediante a análise de componentes principais com rotação varimax forçada a dois fatores (Musitu \& García, 2001), analisando conjuntamente as pontuações sobre o comportamento do pai e as pontuações sobre o comportamento da mãe nas sete práticas parentais do instrumento. Introduziu-se a matriz espanhola de referência - tomada como teórica -, e as obtidas neste estudo em um programa de sintaxe do SPSS para calcular a solução ortogonal Procusto mediante o programa proporcionado por Robert McCrae (veja-se McCrae, Zonderman, P. T. Costa, Bond, \& Paunonen, 1996). O procedimento de Procusto verifica a convergência das estruturas fatoriais comparando os padrões de saturação obtidos ao aplicar um mesmo instrumento (i.e., o ESPA29) com diferentes amostras (pais e mães). Nesta análise calculam-se três Índices de Congruência (IC) entre as amostras: (a) entre as saturações dos itens, (b) entre as subescalas e (c) entre os totais da escala. Quando os índices de congruência são superiores ou iguais a 0,95 , considera-se que mostram uma congruência ótima (McCrae et al., 1996).

\section{Resultados}

\section{Análise Fatorial Exploratória}

Como na normalização espanhola aplicou-se a análise fatorial de componentes principais com rotação varimax, com as pontuações sobre o comportamento da mãe e as pontuações sobre o comportamento do pai, obtendo-se as 2 matrizes fatoriais que se obtiveram na normalização espanhola a partir das 7 subescalas que medem as práticas de socialização utilizadas pela mãe e pelo pai. Esta solução fatorial explicou o 46,27\% do total da variação, o fator I (aceitação/implicação) explicou 25,52\% e o fator 2 (severidade/imposição) 20,75\%. A correlação entre 
as duas dimensões - Aceitação/Implicação e Severidade/ Imposição - foi relativamente ortogonal, $r(n=2089)=$ $0,13, R^{2}<0,02, p<0,01$, com um tamanho do efeito estatisticamente significativo, porém muito pequeno (veja-se García, Pascual, Frías, Van Krunckelsven, \& Murgui, 2008).

Na Tabela 2 comparam-se as matrizes fatoriais da normalização espanhola (Musitu \& García, 2001) com as da adaptação brasileira obtidas no presente estudo, observando-se que, nas amostras dos dois países, o fator Aceitação/Implicação está integrado pelas subescalas Afeto do pai e da mãe e Diálogo do pai e da mãe, que saturam positivamente no fator, e pelas subsescalas de Indiferença e Displicência de ambos os pais que saturam negativamente. Os pesos das subescalas no fator são iguais ou superiores a 0,56 na normalização espanhola e iguais ou superiores a 0,52 na adaptação brasileira, embora as saturações tendam a ser mais elevadas nas análises da normalização espanhola, salvo no caso da subescala Displicência, cujas saturações tanto para as pontuações do comportamento do pai como para o da mãe são mais elevadas nas análises da adaptação brasileira. Por outro lado, enquanto nos dados da normalização espanhola as subescalas que mais alto saturam neste fator são o Afeto e a Indiferença empregadas pela mãe, seguidas do Afeto e a Indiferença empregadas pelo pai; no caso da adaptação brasileira as subescalas que mais alto saturam são a Indiferença por parte da mãe e a Displicência por parte de ambos, pai e mãe, seguidas da Indiferença por parte do pai.
O fator Severidade/Imposição está composto nas duas amostras pelas subescalas Privação, Coerção verbal e Coerção física do pai e da mãe, que saturam positivamente no fator com pontuações iguais ou superiores a 0,61 na normalização espanhola e iguais ou superiores a 0,57 na adaptação brasileira. Ambas, as saturações das subescalas de comportamento do pai e as do comportamento da mãe tendem a ser mais elevadas nos dados da normalização espanhola. A privação por parte do pai e da mãe é a subescala que mais alto satura neste fator, tanto nos dados da normalização espanhola como na adaptação brasileira, seguida de coerção verbal e por último a coerção física, de maneira idêntica nas duas amostras.

\section{Rotação Ortogonal Procusto e Índices de Congruência}

A matriz de saturações fatoriais do presente estudo (Veja a Tabela 2) mostra uma estrutura muito similar à amostra de normalização espanhola. Índices de congruência superiores ou iguais a 0,95 se consideram que mostram uma congruência ótima (McCrae et al., 1996). O índice de congruência do fator Aceitação/Implicação é de 0,98 e o do fator Severidade/Imposição de 0,94 , ligeiramente inferior ao índice ótimo, sendo o índice de congruência total para os dois fatores de 0,96 . Finalmente, só o índice de congruência da subescala diálogo obteve um valor inferior ao ponto de corte, tanto para as pontuações das práticas do pai, 0,88 , como para as da mãe, 0,88 . O resto das subescalas apresenta índices de congruência ótimos.

Tabela 2

Análises de Componentes Principais com Rotação Varimax do ESPA29 com 2 Fatores

\begin{tabular}{lrrrrrrr}
\hline & \multicolumn{2}{c}{$\begin{array}{c}\text { Musitu e García, } \\
\text { 2001 }\end{array}$} & \multicolumn{2}{c}{$\begin{array}{c}\text { Presente } \\
\text { Estudo }\end{array}$} & \multicolumn{3}{c}{$\begin{array}{c}\text { Rotação ortogonal } \\
\text { Procusto }\end{array}$} \\
\cline { 2 - 9 } & $\mathbf{A} / \mathbf{I}$ & $\mathbf{S} / \mathbf{I}$ & $\mathbf{A} / \mathbf{I}$ & $\mathbf{S} / \mathbf{I}$ & $\mathbf{A} / \mathbf{I}$ & $\mathbf{S} / \mathbf{I}$ & $I C$ \\
\hline Mãe & & & & & & & \\
Afeto [Demonstra carinho] & 0,77 & 0,16 & 0,53 & 0,21 & 0,55 & 0,16 & 1,00 \\
Diálogo [Fala comigo] & 0,68 & 0,10 & 0,52 & 0,46 & 0,56 & 0,41 & 0,88 \\
Indiferença [Não se preocupa comigo] & $-0,78$ & 0,08 & $-0,68$ & 0,27 & $-0,65$ & 0,33 & 0,93 \\
Displicência [tanto faz] & $-0,63$ & $-0,02$ & $-0,65$ & 0,11 & $-0,64$ & 0,17 & 0,96 \\
Privação [Retira algo ou proíbe] & 0,13 & 0,80 & $-0,02$ & 0,75 & 0,05 & 0,75 & 1,00 \\
Coerção verbal [Reclama comigo] & 0,14 & 0,74 & 0,21 & 0,62 & 0,27 & 0,60 & 0,97 \\
Coerção física [Bate em mim] & $-0,13$ & 0,62 & $-0,19$ & 0,60 & $-0,13$ & 0,62 & 1,00 \\
Pai & & & & & & & \\
Afeto [Demonstra carinho] & 0,75 & 0,16 & 0,57 & 0,29 & 0,59 & 0,24 & 0,99 \\
Diálogo [Fala comigo] & 0,64 & 0,07 & 0,57 & 0,48 & 0,61 & 0,43 & 0,88 \\
Indiferença [Não se preocupa comigo] & $-0,70$ & 0,13 & $-0,62$ & 0,27 & $-0,59$ & 0,33 & 0,95 \\
Displicência [tanto faz] & $-0,56$ & 0,03 & $-0,65$ & 0,06 & $-0,64$ & 0,12 & 0,99 \\
Privação [Retira algo ou pró́be] & 0,11 & 0,80 & $-0,03$ & 0,77 & 0,04 & 0,77 & 1,00 \\
Coerção verbal [Reclama comigo] & 0,13 & 0,71 & 0,17 & 0,69 & 0,23 & 0,67 & 0,99 \\
Coerção física [Bate em mim] & $-0,14$ & 0,61 & $-0,25$ & 0,57 & $-0,20$ & 0,59 & 1,00 \\
IC & & & & & 0,98 & 0,94 & 0,96 \\
\hline
\end{tabular}

Nota. A/I, Aceitação/Implicação; S/I, Severidade/Imposição; IC, Índice de Congruência.

${ }^{a}$ Dados da normalização espanhola. 


\section{Consistência Interna}

O alfa de Cronbach dos 232 itens foi 0,96; para cada dimensão foram: Aceitação/Implicação, 0,97, e Severidade/Imposição, 0,96; e por cada subescala que mede uma prática parental foram: Afeto, 0,96, Indiferença, 0,95, Diálogo, 0,95, Displicência, 0,90, Coerção verbal, 0,94, Coerção física, 0,93 e Privação, 0,96.

\section{Discussão}

Os resultados obtidos tanto através da análise fatorial de componentes principais quanto da rotação Procusto confirmam, na população brasileira, a estrutura fatorial do ESPA29 obtida na Espanha. Do mesmo modo que nos dados espanhóis, a análise fatorial exploratória mostrou que as subescalas que representam as práticas de Afeto e Diálogo se localizam no pólo positivo da dimensão Aceitação/Implicação, enquanto que as subescalas que representam as práticas opostas - Indiferença e Displicência - localizam-se no pólo negativo desta dimensão. As subescalas que representam as práticas de Privação, Coerção verbal e Coerção física se localizaram na dimensão Severidade/Imposição. Do mesmo modo, a rotação Procusto mostra índices de congruência ótimos com a versão espanhola nas duas dimensões da escala - Aceitação/Implicação e Severidade/Imposição - e em todas as subescalas que medem as práticas parentais, salvo na subescala diálogo, onde a congruência é um pouco menos do que ótima.

Portanto, comprova-se globalmente a estrutura teórica da Escala de Socialização Parental ESPA29 na população brasileira, apoiando a existência de duas dimensões universais e independentes da conduta parental no processo de socialização. Estas duas dimensões refletem dois tipos de padrões persistentes na atuação parental (Musitu \& García, 2001) que ao serem ortogonais, (não estão relacionados e a atuação de um deles não permite conhecer qual será a atuação no outro), precisam ser analisados conjuntamente para determinar o estilo da relação e as implicações que tem cada tipo de relação entre pais e filhos (Darling \& Steinberg, 1993; Smetana, 1995; Steinberg, 2005).

Por outra parte, a operacionalização destas duas dimensões no ESPA29, confirmada neste trabalho no Brasil, é congruente com a utilizada em muitas das medidas que analisam a conduta parental já que, como Steinberg (2005) assinalou, a maior parte dos estudos operacionalizaram uma das dimensões mediante medidas de Severidade e Firmeza parental e a outra mediante medidas de Afeto e Aceitação parental, como por exemplo, as de Firmeza/ Supervisão e Aceitação/Implicação utilizadas por Steinberg e seus colegas (Steinberg et al., 1994), as de Aceitação/Afeto e Controle (García \& Gracia, 2009, 2010; Rohner, 1990) ou as de Aceitação/Implicação e Severidade/Imposição, contempladas no ESPA29 (LópezJáuregui \& Oliden, 2009; Martínez, 2009; Martínez \&
García, 2008; Martínez et al., 2003; Musitu \& García, 2001, 2004).

Contudo, este estudo apresenta ao menos uma limitação que deve ser considerada. Trata-se de uma amostra que, embora tenha um tamanho considerável e similar ao da amostra espanhola, encontra-se constituída por jovens de uma só região do Brasil. Esta especificidade da amostra possivelmente é responsável pelas pequenas variações que ocorrem na subescala diálogo, com respeito à amostra espanhola, descritas anteriormente. Futuras investigações deveriam ampliar a amostragem, utilizando uma amostra representativa de todo o Brasil, de maneira que seja possível a padronização do instrumento no Brasil, como se realizou na Espanha.

Não obstante, apesar dessas limitações, os resultados manifestam claramente que a versão em português brasileiro do instrumento ESPA29 é adequada para medir os estilos de socialização através das dimensões Aceitação/ Implicação e Severidade/Imposição. De modo que se analisem diferentes padrões de relação, persistentes ao longo da relação paterno-filial, nas mesmas situações típicas com diferentes modos de intervenção ou práticas parentais.

\section{Referências}

Arnett, J. J. (1995). Broad and narrow socialization: The family in the context of cultural theory. Journal of Marriage and the Family, 54, 339-373.

Baldwin, A. L. (1955). Behavior and development in childhood. New York: Dryden.

Baumrind, D. (1967). Child cares practices anteceding three patterns of preschool behavior. Genetic Psychology Monographs, 75, 43-88.

Baumrind, D. (1971). Current theories of parental authority. Developmental Psychology Monographs, 4(1, Pt. 2), 1-103.

Baumrind, D. (1991). The influence of parenting style on adolescent competence and substance abuse. Journal of Early Adolescence, 11, 56-94.

Baumrind, D. (1996). The discipline controversy revisited. Family Relations, 4(4), 405-414.

Becker, W. C. (1964). Consequences of different kinds of parental discipline. In M. L. Hoffman \& L. W. Hoffman (Eds.), Review of child development research (Vol. 1, pp. 169208). New York: Russell Sage Foundation.

Brislin, R. W. (1970). Back-translation for cross-cultural research. Journal of Cross-Cultural Psychology, 1, 185-216.

Camino, C., Camino, L., \& Moraes, R. (2003). Moralidade e socialização: Estudos empiricos sobre práticas maternas de controle social e o julgamento moral [Morality and socialization: Empirical studies on maternal practices of social control and the moral judgment]. Psicologia: Reflexão e Critica, 16, 41-61.

Chao, R. K. (2001). Extending research on the consequences of parenting style for Chinese Americans and European Americans. Child Development, 72, 1832-1843.

Costa, F. T., Teixeira, M. A. P., \& Gomes, W. B. (2000). Responsividade e exigência: Duas escalas para avaliar estilos parentais [Responsiveness and demandingness: Two scales to measure parenting styles]. Psicologia: Reflexão e Crítica, $13,465-473$. 
Darling, N., \& Steinberg, L. (1993). Parenting style as context: An integrative model. Psychological Bulletin, 113, 487-496.

García, F., \& Gracia, E. (2009). Is always authoritative the optimum parenting style? Evidence from Spanish families. Adolescence, 44(173), 101-131.

García, F., \& Gracia, E. (2010). ¿Qué estilo de socialización parental es el idóneo en España? Un estudio con niños y adolescentes de 10 a 14 años [What is the optimum parenting style in Spain? A study with children and adolescents aged 10-14]. Infancia y Aprendizaje, 33, 365-384.

García, J. F., Musitu, G., \& Veiga, F. (2006) Autoconcepto en adultos de España y Portugal [Self-concept in adults from Spain and Portugal]. Psicothema, 18, 551-556.

García, J. F., Pascual, J., Frías, M. D., Van Krunckelsven, D., \& Murgui, S. (2008). Diseño y análisis de la potencia: $n$ y los intervalos de confianza de las medias [Design and power analysis: $n$ and confidence intervals of means]. Psicothema, 20, 933-938.

Gray, M. R., \& Steinberg, L. (1999). Unpacking authoritative parenting: Reassessing a multidimensional construct. Journal of Marriage and the Family, 61, 574-587.

Grusec, J. E., \& Lytton, H. (1988). Social development: History, theory, and research. New York: Springer-Verlag.

Kremers, S. P., Brug, J., de Vries, H., \& Engels, R. C. M. E. (2003). Parenting style and adolescent fruit consumption. Appetite, 41, 43-50.

Lamborn, S. D., Mounts, N. S., Steinberg, L., \& Dornbusch, S. M. (1991). Patterns of competence and adjustment among adolescents from authoritative, authoritarian, indulgent, and neglectful families. Child Development, 62, 1049-1065.

López-Jáuregui, A., \& Oliden, P. E. (2009). Adaptation of the ESPA29 Parental Socialization Styles Scale to the Basque language: Evidence of validity. Spanish Journal of Psychology, 12, 737-745.

Maccoby, E. E., \& Martin, J. A. (1983). Socialization in the context of the family: Parent-child interaction. In P. H. Mussen (Ed.), Handbook of child psychology (Vol. 4, pp. 1101). New York: Wiley.

Marchetti, B. (1997). Concetto di se'relazioni familiari e valori [Relations between family and value concepts]. Unpublished master's thesis, University of Bologna, Italy.

Martínez, I. (2003). Estudio transcultural de los estilos de socialización parental [A cross cultural analysis of parenting]. Unpublished doctoral dissertation, University of Valencia, Spain.

Martínez, I. (2008). Repercusiones de la utilización del castigo fiisico sobre los hijos: Influencia del contexto familiar [The impact of corporal punishment on children: Influence of family environment]. Psicología Educativa, 14, 91-102.

Martínez, I. (2009). Influence of parenting style on Schwartz adolescents'values from Spain and Brazil. Paper presented at the 11th European Congress of Psychology, Oslo, Norway.

Martínez, I., \& García, J. F. (2007). Impact of parenting styles on adolescents' self-esteem and internalization of values in Spain. Spanish Journal of Psychology, 10, 338-348.

Martínez, I., \& García, J. F. (2008). Internalization of values and self-esteem among Brazilian teenagers from authoritative, indulgent, authoritarian, and neglectful homes. Adolescence, 43(169), 13-29.

Martínez, I., García, J. F., \& Yubero, S. (2007). Parenting styles and adolescents' self-esteem in Brazil. Psychological Reports, 100, 731-745.
Martínez, I., \& Madrid, I. (2008). La utilización de prácticas coercitivas por parte de los padres en España y Brasil: un estudio comparativo [Use of coercitive practices by parents in Spain and Brazil: A comparative study]. Psicologia, Educação e Cultura, 12, 283-294.

Martínez, I., Musitu, G., García, J. F., \& Camino, L. (2003). Un análisis intercultural de los efectos de la socialización familiar en el autoconcepto: España y Brasil [A cross-cultural analysis of the effects of family socialization on self-concept: Spain and Brazil]. Psicologia, Educação e Cultura, 7, 239259.

Martínez, I., \& Navarro, R. (2008). The influence of parental child-rearing practices on Spanish adolescent values [Special issue]. International Journal of Psychology, 3-4, 56-56.

McCrae, R. R., Zonderman, A. B., Bond, M. H., Costa, P. T., \& Paunonen, S. V. (1996). Evaluating replicability of factors in the revised NEO personality inventory: Confirmatory factor analysis versus procrustes rotation. Journal of Personality and Social Psychology, 70, 552-566.

Mischel, W., \& Mischel, H. N. (1976). A cognitive sociallearning approach to morality and self-regulation. In T. Lickona (Ed.), Moral development and behavior: Theory, research, and social issues (pp. 84-107). New York: Holt, Rinehart, \& Winston.

Moraes, R., Camino, C., Costa, J. B., Camino, L., \& Cruz, L. (2007). Socialização parental e valores: Um estudo com adolescentes [Parental socialization and values: A study with teenagers]. Psicologia: Reflexão e Crítica, 20, 167-177.

Musitu, G., \& García, F. (2001). ESPA29: Escala de estilos de socialización parental en la adolescencia [ESPA29: Parental socialization scale in adolescence]. Madrid, Spain: Tea.

Musitu, G., \& García, J. F. (2004). Consecuencias de la socialización familiar en la cultura española [Consequences of the family socialization in the Spanish culture] Psicothema, 16, 288-293.

Oliva, A. (2006). Relaciones familiares y desarrollo adolescente [Family relationships and adolescent development]. Anuario de Psicología, 37, 209-223.

Oliveira, E. A. F., Marin, A. H., Pires, F. B., Frizzo, G. B., Ravanello, T., \& Rossato, C. (2002). Estilos parentais autoritário e democrático-recíproco intergeracionais, conflito conjugal e comportamentos de externalização e internalização [Intergenerational authoritarian and democratic-reciprocal parenting styles, marital conflict and externalizing and internalizing behaviors]. Psicologia Reflexão e Crítica, 15, $1-11$.

Parke, R. D. (1974). Rules, roles, and resistance to deviation: Recent advances in punishment, discipline, and self-controld self-regulation. In A. D. Pick (Ed.), Minnesota Symposium on Child Psychology (Vol. 8, pp. 111-143). Minneapolis, MB: University of Minnesota Press.

Rohner, R. P. (1990). Handbook for the study of parental acceptance and rejection ( $3^{\text {rd }}$ ed.). Storrs, CT: Rohner Research Publications.

Scarr, S. (1993). Biological and cultural diversity: The legacy of Darwin for development. Child Development, 64, 13331353.

Schaefer, E. S. (1959). A circumplex model for maternal behavior. Journal of Abnormal and Social Psychology, 59, 226-235.

Sears, R. R., Maccoby, E. E., \& Levin, H. (1957). Patterns of child rearing. Evanston, IL: Row, Peterson. 
Smetana, J. G. (1995). Parenting styles and conceptions of parental authority during adolescence. Child Development, 66, 299-316.

Steinberg, L. (2005). Psychological control: Style or substance? New Directions for Child and Adolescent Development, 108, 71-78.

Steinberg, L., Lamborn, S. D., Darling, N., Mounts, N. S., \& Dornbusch, S. M. (1994). Over-Time changes in adjustment and competence among adolescents from authoritative, authoritarian, indulgent, and neglectful families. Child Development, 65, 754-770.

Symonds, P. M. (1939). The psychology of parent-child relationships. New York: Appleton-Century-Crofts.

Villalobos, J. A., Cruz, A. V., \& Sánchez, P. R. (2004). Estilos parentales y desarrollo psicosocial en estudiantes de Bachillerato [Parental styles and psychosocial development in High-School students]. Revista Mexicana de Psicología, 21, 119-129.

Weber, L. N. D., Prado, P. M., Viezzer, A. P., \& Brandenburg, O. J. (2004). Identificação de estilos parentais: O ponto de vista dos pais e dos filhos [Identifying parenting styles: Parent's and children's point of view]. Psicologia: Reflexão e Crítica, 17, 323-331. 